\title{
Comparative Analysis of Clustering Protocols for Wireless Sensor Networks
}

\author{
Harpinder Kaur \\ Department of Computer \\ Science \\ Guru Nanak Dev University, \\ Amritsar
}

\author{
Navjot Kaur \\ Department of Computer \\ Science \\ Guru Nanak Dev University, \\ Amritsar
}

\author{
Sandeep Waraich \\ Department of computer \\ Science \\ Guru Nanak Dev University, \\ Amritsar
}

\begin{abstract}
Wireless Sensor networks include large amount of low power, low priced sensor nodes generally deployed in hostile and harsh environment to sense, collect and transmit data to sink at far distance. Clustering has been widely studied to enhance the lifetime of WSN by reducing the number packet transmission. In clustering, the nodes selected as cluster head, often suffer from high overload and thus consume more energy. Re-clustering is eventually performed to talk about the resource intensive cluster head role, which requires global time synchronization. To handle this problem, some recent research has been considered in the field of the wireless sensor networks. The overall objective is to judge the various limitations of the sooner techniques. This paper ends up with the suitable future directions to boost the existing protocol further.
\end{abstract}

\section{Keywords}

WSNs, Energy Efficiency, Clustering, Sensing.

\section{INTRODUCTION}

A WSN is typically consists of many sensor nodes to sense the physical characteristics of the world. The motivation of development of WSN was military applications such as battlefield surveillance, HHTI (Handheld thermal imager). Nowadays these networks have expended their scope in many industrial and consumer applications, i.e. industrial process monitoring and control, machine health monitoring and so on [1]. The WSN consists of nodes that may vary from few to several hundreds or thousands, where each node is connected to one or more sensors.

The enormous growth in usage of WSN in the last decade leads to the crucial need for scalable, energy-efficient, data gathering and aggregation protocols in corresponding largescale environments. This can be achieved by hierarchical, each cluster has a cluster head $(\mathrm{CH})$ and usually performs the special tasks such as data fusion and aggregation, and several common sensor nodes known as member nodes (MN). A twolevel hierarchical structure is obtained from the cluster formation process, where the $\mathrm{CH}$ nodes form the higher level and the cluster-member nodes form the lower level [2].

The $\mathrm{CH}$ nodes spend energy at higher rates than MNs as these send all the time data to higher distances than the member nodes. In order to balance the energy consumption among all the network nodes, a common solution is to periodically reelect new $\mathrm{CHs}$ in each cluster.

Before documenting on the possible classification options of WSNs clustering algorithms, it is significant to clarify some important parameters regarding the whole clustering procedure in WSNs.

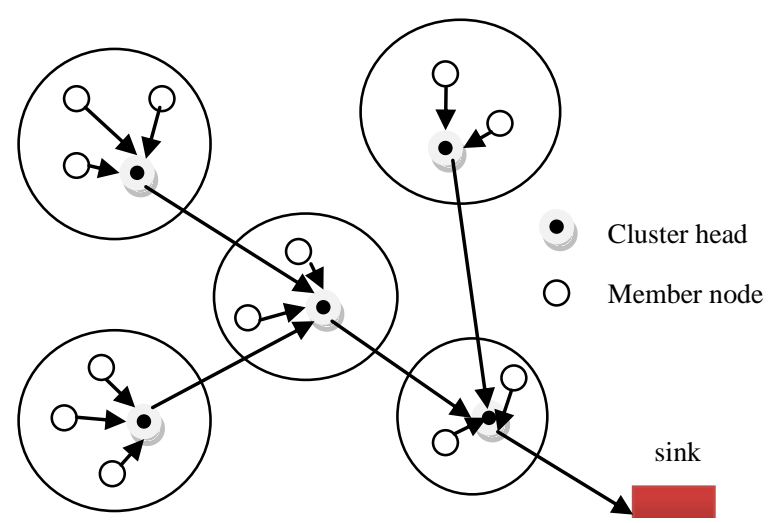

Figure 1: Data communication in clustered network

Moreover these parameters are served as the basic means for comparison and categorization of various clustering protocols discussed in this paper.

i. Number of clusters (cluster count): In most recent probabilistic and randomized clustering algorithms the $\mathrm{CH}$ election and formation process lead naturally to variable number of clusters. In some published approaches, however, the set of $\mathrm{CHs}$ are predetermined and thus the numbers of clusters are preset. The amount of clusters is generally a critical parameter pertaining to the efficiency of the total routing protocol.

ii. Intra-cluster communication: In some initial clustering approaches the communication between a sensor and its designated $\mathrm{CH}$ is assumed to be direct. However, multi-hop intra-cluster communication is often required, i.e., when the communication range of the sensor nodes is limited or the number of sensor nodes is very large and the number of $\mathrm{CHs}$ is bounded.

iii. Cluster formation methodology: In most recent approaches, when $\mathrm{CHs}$ are just regular sensors nodes and time efficiency is a primary design criterion, clustering is being performed in a distributed manner without coordination. In few earlier approaches a centralized (or hybrid) approach is followed; one or more coordinator nodes are used to partition the whole network off-line and control the cluster membership.

iv. Cluster-head selection: The cluster heads of the clusters in some proposed algorithms (mainly for heterogeneous environments) can be pre-assigned. Generally however in homogeneous environments, the $\mathrm{CHs}$ are picked from the deployed set of nodes either in a probabilistic or completely 
random way or based on other more specific criteria (residual energy, connectivity etc.).

v. Algorithm complexity: In most recent algorithms the fast termination of the executed protocol is one of the primary design goals. Thus, the time complexity or convergence rate of most cluster formation procedures proposed nowadays is constant (or just dependent on the amount of $\mathrm{CHs}$ or the amount of hops). In some earlier protocols, however, the complexity time has been allowed to depend on the total number of sensors in the network, focusing in other criteria first.

vi. Multiple levels: In several published approaches the idea of a multi-level cluster hierarchy is introduced to achieve even better energy distribution and total energy consumption (instead of using only one cluster level). The improvements offered by multi-level clustering can be further studied, especially when we have very large networks and inter-CH communication efficiency is of high importance.

vii. Overlapping: Several protocols give also high importance on the idea of node overlapping within different clusters (either for better routing efficiency or for faster cluster formation protocol execution or for other reasons).

\section{RELATED WORK}

Node clustering in WSNs has been extensively studied before decade, with several protocols proposed to save limited sensor energy in order to extend network lifetime. The initial generations of clustering protocols are designed for networks with direct links between CHs and data sink. The LEACH protocol provides the initial exemplary instance of how a WSN may be autonomously organized to give its lifetime. To get this done, the strategy is to equalize the full time fraction that different nodes take force intensive $\mathrm{CH}$ role. Beginning with equal vitality, each node picks a probability value to decide whether to behave as a $\mathrm{CH}$, on the foundation of the amount of times it has claimed the $\mathrm{CH}$ role. An improved LEACH, called M-LEACH has been proposed, in which nodes in a cluster deliver data to the $\mathrm{CH}$ over multiple paths and can thus achieve energy savings. Similarly, k-hop forwarding from cluster members towards the $\mathrm{CH}$ is included in [9] with simpler sensors that have a fixed radius rather than an adjustable one.

Rather than the received signal strength (RSS) from neighbor $\mathrm{CHs}$, later clustering protocols introduce different matrix for sensors for making a decision on which $\mathrm{CH}$ to join. EECH [1] proposes awaited cost function that takes into account sensor to $\mathrm{CH}$ and $\mathrm{CH}$ to sink distances to consider the transmission costs. HEED [4] introduces average reachability power, which is the average minimum transmission power to reach a $\mathrm{CH}$ from its neighbor nodes. This is used as an estimate of the prospective intra-cluster communication costs if the node chooses to act as a $\mathrm{CH}$.

Residual node energy levels have been considered in clustering protocols to assign the $\mathrm{CH}$ role to resource rich sensors. EEHC [1] is one example, which is made for networks with direct links to the data sink from $\mathrm{CH}$. Similarly, HEED [4] takes into account residual node energy levels and uses $\mathrm{CH}$ node degrees to uniformly distribute the $\mathrm{CH}$ in the network and provide high connectivity. Similar to HEED, DEEC can provide fairer $\mathrm{CH}$ election in relation to energy levels, but all nodes need to know the newest average node energy in the network. An improvement of HEED is VCA protocol which uses a voting mechanism to elect the $\mathrm{CHs}$ and regular neighborhood messages to inform neighbor node of residual energy levels.

A part from sensors residual battery energy, a high traffic load is another factor that leads to early energy depletion. Towards this, some approaches [11] have consider the imbalance in traffic load between different sensors, and as remedy, clusters of different sizes are formed i.e. the $\mathrm{CH}$ communication range with cluster members. UCS considers powerful nodes predeployed in the network to serve as $\mathrm{CHs}$, where as other UCR and $\mathrm{EC}$ [11], elect $\mathrm{CH}$ in regular time rounds. These protocols are aimed at improving network life time via. Eliminating network hotspot caused by the imbalance in traffic loads on $\mathrm{CH}$ due to multihop forwarding to the data center. UCR needs to estimate internode distances using RSS levels to adjust node transmission ranges. In Comparison, EC can provide longer network lifetime; however it relies on estimation of end to end packet delivery energy costs in a specific rectangular topology i.e. divided into virtual regions.

The common mechanism in node clustering in WSNs is that network runtime is divided into time rounds which start with a short re-clustering stage followed by a longer data transmission stage. To manage to rotate the load intensive $\mathrm{CH}$ role, nodes periodically perform re-clustering operations at each re-clustering stage to elect a new set of $\mathrm{CHs}$ that will assist the network during the next round. Recently, some studies have emphasized the node for a fully asynchronous operation in fully self-organized WSN, which does not required time rounds. These asynchronous node clustering protocols are designed for networks with single hops from $\mathrm{CHs}$ to data sink and are not applicable to multihop traffic loads.

\section{CLUSTERING PROTOCOLS}

Number of clustering algorithms has been proposed for prolonging the life of WSN. We have reviewed a few of the clustering techniques used in literature and categorized them as synchronous and asynchronous protocols on the basis of $\mathrm{CH}$ selection criterion.

\subsection{Synchronous Clustering Protocols}

Protocols based on time-round require network wide time synchronization so that in re-clustering phase, nodes can simultaneously engage in and perform cooperative operations to elect the new set of $\mathrm{CHs}$. The cluster formation procedure here includes the communication of nodes with their neighbors and requires more intensive exchange of messages and probably graphs traversing in some extent. However, in synchronous clustering protocols, $\mathrm{CH}$ rotation is carried out frequently in the entire network. The large overhead occurs as every time clusters are reformed. Also none of the existing algorithms in this class provide the optimal value of the period or data rounds. A few of the algorithms that fall under this category are discussed below.

\subsubsection{Low Energy Adaptive Clustering Hierarchy (LEACH)}

The first clustering protocol proposed for WSNs was LEACH. It is a dynamic, hierarchical, probabilistic, distributed and one-hop protocol. Its major objectives are to improve the lifetime of WSNs by evenly distributing the energy consumption among all the nodes of the network and to reduce the energy consumption in the network nodes by performing data aggregation. The cluster formation is done on the basis of the received signal strength and the $\mathrm{CH}$ nodes act as routers to the BS. All the data processing i.e. data fusion and aggregation are local to the cluster. 
In $\mathrm{LEACH}$, load balancing is achieved by $\mathrm{CH}$ role rotation which is performed periodically among the nodes of the cluster. Each node chooses a random number "T" between 0 and 1 to perform cluster head rotation. A node is considered as $\mathrm{CH}$ for the given round if the number is less than the following threshold:

$\mathrm{T}(\mathrm{i})=\left\{\begin{array}{c}\frac{p}{1-p *\left(r \bmod \frac{1}{p}\right)} \\ 0 \quad \text { otherwise }\end{array} \quad\right.$ if $i \in G$

where

$p$ is the desired percentage of $\mathrm{CH}$ nodes in the sensor population

$r$ is the current round number

$G$ is the set of nodes that have not been $\mathrm{CHs}$ in the last $1 / p$ rounds

The clusters are formed dynamically in each round through the process given in Figure 2 and the time to perform each round is also selected randomly. Generally, in one-hop sensor networks, LEACH can provide a quite uniform load distribution and Furthermore, the localized coordination scheme used in LEACH provides better scalability for cluster formation.

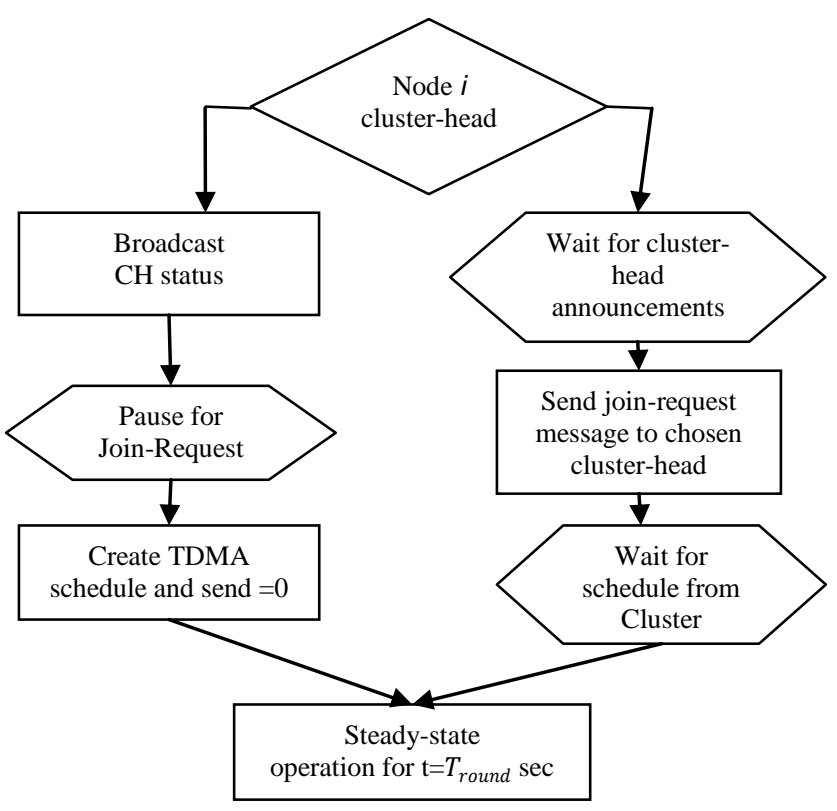

Figure: 2 The cluster formation process of LEACH

Despite the good performance, LEACH also has some flaws. Because of probabilistic criterion, good $\mathrm{CHs}$ distribution cannot be guaranteed and some nodes will not have any $\mathrm{CH}$ in their range. The $\mathrm{CHs}$ are assumed to have a long communication range so that the data can reach the BS directly which is simply not always a realistic assumption Additionally, LEACH is applicable in one-hop intra-cluster and inter-cluster topology thus it cannot be used effectively on large scale networks.

\subsubsection{LEACH-F}

It is an improved version of LEACH protocol that includes fixed clusters and rotating cluster heads [22]. The clusters are formed once and fixed, and within the cluster, the clusterhead's position rotates among the nodes. There is no set-up overhead, since clusters are formed only once in the beginning of each round. It does not allow to add new nodes to the system and do not adjust their behavior on the basis of nodes dying.

\subsubsection{LEACH-C}

W. B. Heinzelman et al. [23] has given application specific protocol architecture for WSN which is known as LEACH Centralized. LEACH-C is an improved version of LEACH. A centralized clustering algorithm is used in it and the similar steady-state phase as that used in LEACH. LEACH-C delivers about $40 \%$ more data per unit energy than LEACH, so LEACH-C is considered as more efficient than LEACH.

\subsubsection{E-LEACH}

The $\mathrm{CH}$ selection procedure in LEACH is improved in energy-LEACH [24]. Residual energy of node is taken as the main metric which decides whether the nodes are chosen as $\mathrm{CH}$ or not after the first round. The operation of E-LEACH is divided into rounds; every node has the same probability to turn into $\mathrm{CH}$ in the initial round, that mean $\mathrm{CHs}$ are formed randomly. In the next rounds, after one round communication, the residual energy of each node is different and is considered for the selection of the CHs. Thus, nodes with more energy will become $\mathrm{CHs}$ rather than nodes with less energy.

\subsubsection{V-LEACH}

V-LEACH [24] is an enhancement in LEACH Protocol in order to reduce energy consumption. The main concept behind V-LEACH is to have a vice-CH besides having a $\mathrm{CH}$ in the cluster, which takes the role of the $\mathrm{CH}$ when the $\mathrm{CH}$ dies. This idea made possible that cluster nodes data will always reach the $\mathrm{BS}$ and hence eliminated the need to elect a new $\mathrm{CH}$ each time the $\mathrm{CH}$ dies which will extend the overall network life time.

\subsubsection{H-LEACH}

Hierarchical LEACH (H-LEACH) is given by Wairagu G. Richard considering the concept of energy conservation by minimizing the communication distance between nodes. The same clustering approach is employed during initial phases as LEACH and by further clustering the cluster heads, extension is done on LEACH and one of the cluster head is nominated which acts as the Master Cluster Head (MCH) to forward data to the base station. Since, only one $\mathrm{MCH}$ is involved to transmit all compressed data to base station, so when the $\mathrm{MCH}$ will be dead, central point of failure situation may occur.

\subsubsection{Two-Level Hierarchy LEACH (TL- LEACH)}

(TL-LEACH) introduced by Loscrìet al.[6], is an extension to the LEACH protocol. TL-LEACH uses randomized, adaptive, self-configuring cluster formation and localized control for data transfers to achieve energy and latency efficiency. In TL$\mathrm{LEACH}$, a $\mathrm{CH}$ collects data from member nodes but unlike LEACH, instead of transmitting data to the BS directly, it uses an integral part of $\mathrm{CH}$ s that lies between the $\mathrm{CH}$ and the $\mathrm{BS}$ as a relay station. In TL-LEACH two-level hierarchy is introduced i.e. top $\mathrm{CHs}$ called primary cluster heads and secondary cluster heads represents second level. The algorithm consists of four phases: advertisement phase, cluster setup phase, schedule creation and data transmission.

\subsubsection{Power-Efficient Gathering in Sensor Information Systems (PEGASIS)}

PEGASIS, given by Lindsey et al. [7], is an improvement of LEACH. Every sensor in the network transmits its data to one of its neighbors. In this way, the gathered data is transferred 
from one node to another through a chain, which can either be concentratedly assigned by the sink and broadcast to all nodes or accomplished by the nodes themselves. If the chain is formed by the nodes themselves, they can first get the location data of all nodes and locally compute the chain using the greedy algorithm. The chain construction is commenced from the furthest node from the sink and the closest neighbor to this node would be the next node on the chain. When a node on the chain dies, the chain will be reconstructed in the same manner to bypass the dead node. A designated node sends the assembled data back to the BS. PEGASIS is claimed as a distributed algorithm with even energy load distribution. However, every single node needs to obtain the global topology of the network. In PEGASIS, the locations of nodes are random, and each sensor node has the ability of data detection, wireless communication, data fusion and positioning.

\subsubsection{Hierarchical Geographic Multicast Routing (HGMR)}

HGMR, given in [9] by Koutsonikolas et al., is a locationbased multicast protocol. This protocol incorporates the key design concepts of both the Geographic Multicast Routing and Hierarchical Rendezvous Point Multicast protocols, and provides forwarding energy efficiency along with scalability to large-scale WSNs as an improvement to them. HGMR uses the key concept of mobile geographic hashing for hierarchical decomposition of a multicast group into subgroup of manageable size. Within each subgroup, the local multicast scheme of GMR is adopted to forward data packets along multiple branches of the multicast tree in one transmission. In mobile geographic hashing, the deployment area is recursively partitioned into numerous $d 2$ equal-sized square sub-domains called cells, where $d$ is decomposition index with respect to the encoding overhead constraints, and each cell comprises a manageably-sized subgroup of members controlled by Access Point (AP) and a Rendezvous Point (RP)is used to manage all APs.

\subsubsection{Unequally Clustered Multihop Routing (UCMR) protocol}

Most clustering algorithm provides an equal cluster size using node's ID degree and etc. In [11] a simple clustering algorithm named as Unequally Clustered Multihop Routing (UCMR) protocol is given where each cluster has a different cluster size, based on its distance with reference to base station. Fig. 3 gives model of the unequal clustering network with multihop transmission.

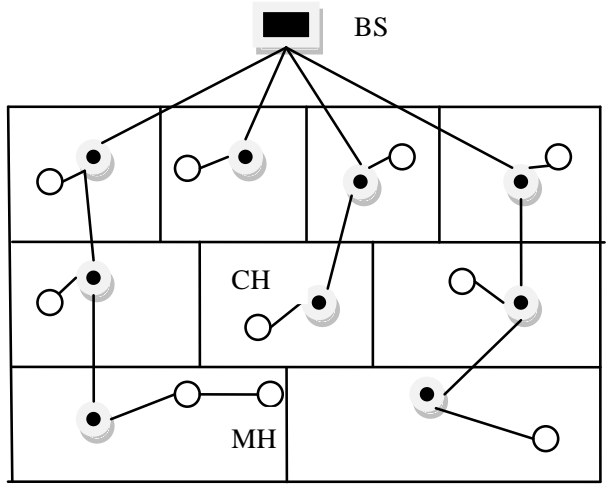

Figure: 3 Overview of UCMR protocol
The size of the cluster is increasing with the distance from base station increases. In order to minimize the energy consumption of network and to improve the overall network performance the multi-hop routing with using Dijkstra's shortest path algorithm is used for intra-cluster and intercluster transmission. The reason to increase life time of network is better positioning of the cluster heads. After the first node dead the energy is more evenly distributed than LEACH and UCR protocols. In [10], it is shown that energy consumption of a node per round in UCMR is lesser than UCR and LEACH. So UCMR algorithm is simple as well as it uses various parameters such as remaining energy, number of neighbor nodes and position of sensors to select $\mathrm{CH}$ which in turn enhances the network lifetime.

\subsubsection{Threshold sensitive Energy Efficient sensor Network protocol (TEEN)}

TEEN [8], introduced by Anjeshwar and Agrawal, is a hierarchical protocol whose key objective is to cope with sudden changes in the sensed attributes. TEEN protocol hybrids the hierarchical technique with a data-centric approach. The nodes sense their environment regularly, since the data transmission is done less frequently therefore, the energy consumption can potentially be much less than that in the proactive network. This protocol uses 2-tier clustering topology and two thresholds i.e. hard threshold and soft threshold. The hard threshold is the absolute value of a sensed attribute such as temperature beyond which, the node sensing this value must switch on its transmitter and report to its $\mathrm{CH}$. Whereas, the soft threshold is a small change in the value of the sensed attribute which stimulate the node to switch on its transmitter and transmit. The hard threshold allows the nodes to transmit only when the sensed attribute is in the range of interest thus, tries to reduce data communications. The soft threshold further reduces data communications. A smaller value of the soft threshold generates more accurate information of the network at the expense of increased energy consumption, thus by the parameters adjustment, users can control the trade-off between energy efficiency and data accuracy. Moreover, the soft threshold can be varied and the users can change the fresh parameters according to every cluster change time.

\subsubsection{Hierarchical Control Clustering-HCC}

HCC [27] is a distributed multi-hop hierarchical clustering algorithm which also efficiently extends to form a multi-level cluster hierarchy. Any node in the WSN can initiate the cluster formation process. The algorithm proceeds in two phases, "Tree Discovery" and "Cluster Formation." The tree discovery phase is basically a distributed formation of a Breadth-First-Search (BFS) tree rooted at the initiator node.

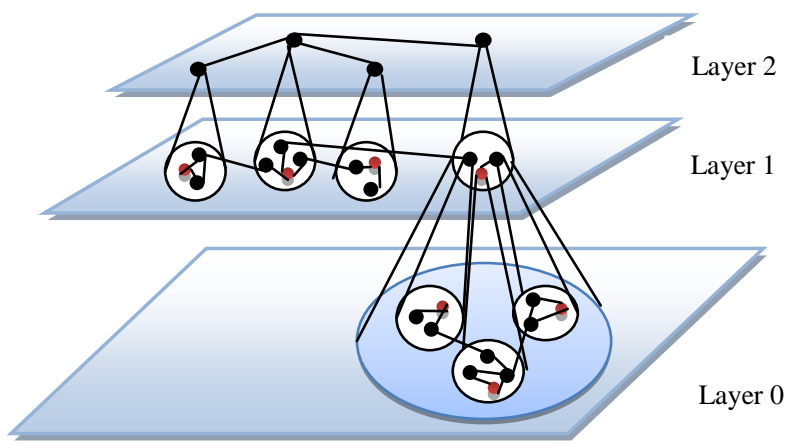

Figure 4: HCC Three-layer cluster hierarchy 
Each node, $\mathrm{u}$, broadcasts a signal once every $p$ units of time, carrying the information about its shortest hop distance to the root, $r$. A node $v$ that is neighbor of $u$ will choose $u$ to be its parent and will update its hop distance to the root, if the route through $u$ is shorter. The broadcast signal carries the parent ID, the root ID, and the sub tree size. Every node updates its sub tree size when its children sub tree size change. The cluster formation phase starts when a sub tree on a node crosses the size parameter, $k$. The node initiates cluster formation on its sub tree. It will form a single cluster for the entire sub tree if the sub tree size is less than $2 k$, or else, it will form multiple clusters. The cluster size and the degree of overlap are also considered.

In Figure 4 the proposed multi-level hierarchy is further illustrated. This approach has a time complexity of $O(n)$, however it has been shown to achieve quite balanced clustering as well as to handle dynamic environments very well.

\subsubsection{Clustering Algorithm via Waiting Timer- CAWT}

CAWT [28], a distributed proximity-connectivity-based algorithm for constructing cluster hierarchy has been proposed for homogeneous sensors with the same transmission range. Once sensors are deployed, each sensor broadcasts a "hello" message to show its presence to the neighbors while listening to the others. The sensors that hear a significant number of "hello" messages (meaning that are nodes with high connectivity) organize into clusters while others are waiting to form clusters. The performance of the algorithm was evaluated using simplified simulations leading to quite good results with regard to network lifetime. However, as it is clearly observed, the generalization of the algorithm is subject to detailed evaluation regarding load balancing, $\mathrm{CH}$ reelection, and energy usage across the network.

\subsubsection{EACLE: Energy-aware clustering scheme with transmission power control}

Similarly, in [26] (EACLE) a distributed clustering procedure, which beyond the proximity takes also in account the residual energy of each node, is followed. It is mainly based on the information of 2-hop neighbors with a practical transmission power control scheme, and then builds a broadcast tree only by cluster heads. Initially, each sensor is in a 'waiting' state and waits for time $T 1$ which really is a monotonous decreasing function on the residual energy of the node. When the timer expires, the waiting node becomes a $\mathrm{CH}$ and broadcasts two packets with different (power-high and powerlow) transmission power each, which contain the list of the neighbor-IDs received before broadcasting. When a waiting node receives a power-low packet it becomes a member node, whereas when it receives a power-high packet, it compares its own neighbor list with the list of IDs in the receiving packet, to decide if it should continue waiting or become a $\mathrm{CH}$. Also, each node executes the clustering process periodically. Once a node becomes a $\mathrm{CH}$ in a specific round, its timer is then set to a longer value to avoid becoming a $\mathrm{CH}$ again in the next round.

\subsubsection{Algorithm for Cluster Establishment-ACE}

A quite valuable alternative approach was given in [29].Unlike other distributed clustering schemes, ACE employs an emergent algorithm. Emergent algorithms much like artificial neural networks evolve to optimal solution through a mix of local optimization steps. Initially, a node decides to become a "candidate" $\mathrm{CH}$, and then it broadcasts an invitation message. Upon getting the invitation, a neighboring sensor joins the new cluster and becomes a follower of the new $\mathrm{CH}$. At any moment, a node could be a follower of more than one cluster. Next, the migration phase takes place in order the best candidate for being $\mathrm{CH}$ to be selected. Each $\mathrm{CH}$ periodically checks the ability of its neighbors for being a $\mathrm{CH}$ and decides to step down if one of these neighbors has more followers than it does. A node that has the largest number of followers and the least overlap with other clusters will be considered as the best final candidate for $\mathrm{CH}$. The algorithm converges in time $O(d)$ where $\mathrm{d}$ is the node density per unit disk. Experimental validation of ACE indicated that it achieves low variance and high average of cluster sizes when comparing to node-ID-based schemes.

\subsection{Asynchronous Clustering Protocols}

Recent studies have proposed asynchronous clustering to avoid the requirement of synchronization. In energy driven clustering algorithms, the $\mathrm{CHs}$ roles are rotated when the residual energy of $\mathrm{CH}$ is less than a threshold value. This method can be used in the global re-clustering as well as in local re-clustering. Thus, the large cost of overheads can be eliminated with this method and also it can prolong the network lifetime.

\subsubsection{Hybrid Energy-Efficient Distributed clustering (HEED)}

HEED [4], proposed by Younis and Fahmy, is a multi-hop WSN clustering algorithm which brings an energy-efficient clustering routing with explicit concern of energy. HEED [4] can select the sensors with high battery level to be $\mathrm{CHs}$ through the iteration $\mathrm{CH}$ election scheme. In the iteration $\mathrm{CH}$ election scheme, $\mathrm{CHs}$ are classified into two as tentative and final $\mathrm{CHs}$. Some initial $\mathrm{CHs}$ are selected randomly as tentative CHs. After each iteration, every sensor increases their probability to become a $\mathrm{CH}$. Once the probability is over 1 , the sensor will claim itself as a final $\mathrm{CH}$. A tentative $\mathrm{CH}$ will give up to be a $\mathrm{CH}$ if it finds a final $\mathrm{CH}$ in its communication range. The sensors are not covered by any final $\mathrm{CHs}$ will elect themselves to be $\mathrm{CHs}$. This iteration $\mathrm{CH}$ election scheme guarantees that the probability of the phenomena that two sensors, within each other's communication range, both become $\mathrm{CHs}$ is rare. Therefore it is can be deduced that the $\mathrm{CHs}$ are well distributed over the network and thus all the clusters have similar size. HEED involves significant overhead due to the heavy broadcast in each iteration. The overhead and network delay are increased as the number of iterations required in the initial phase increases. Unlike LEACH, the HEED does not select nodes as CHs randomly. The manner of cluster construction is performed based on the hybrid combination of two parameters i.e. residual energy and intra cluster communication cost. In HEED, the nodes with average high residual energy are elected as $\mathrm{CHs}$ Additionally one of the major goals of HEED is to get an even distributed $\mathrm{CHs}$ throughout the networks.

\subsubsection{Distributed Weight-based Energy-efficient Hierarchical Clustering protocol (DWEHC)}

DWEHC, given by Ding et al.[5], is a distributed clustering algorithm that provide improvement to HEED by building balanced cluster sizes and optimize the intra-cluster topology using location awareness of the nodes. DWEHC include no assumption about network size and density, and consider residual energy in the act of $\mathrm{CH}$ election as in HEED. Every node individually implements DWEHC and after several iterations that are implemented in a distributed manner, the algorithm ends. Unlike LEACH and HEED, a multi-level 
structure for intra-cluster communication is created in DWEHC and limits number of children of a parent node. Moreover, for $\mathrm{CH}$ election, the only locally calculated parameter weight is defined.

\subsubsection{Cluster based energy efficient routing algorithm (CBER)}

CBER has been proposed in [10], CBER elects $\mathrm{CH}$ based on nodes near to the optimal cluster head distance and residual energy of the nodes. In WSNs energy is mostly consumed for transmission and reception, it is a non linear function of transmission range. In this paper, the optimal cluster head distance which links to optimal energy consumption is derived. In addition, residual energy is considered in the $\mathrm{CH}$ election in order to increase the network lifetime. Furthermore, the energy consumption of being a $\mathrm{CH}$ is equally spread among the cluster members. Performance results show CBER scheme reduces the end to end energy consumption and prolong the network lifetime of multi hop network compared to the well-known clustering algorithms LEACH and HEED.

\subsubsection{Hop distances optimization for balancing the energy consumption of multi-hop clustered Wireless sensor Networks}

One solution for the hot-spot problem of multi-hop communication, is designing an unequal cluster model [12]. Some algorithms have been proposed to realize it, but just a couple of that gives a comprehensive energy model. In [12], a new scheme for the optimum partitioning area is presented. The scheme is dependent on a circular form as shown in Fig. 4. In this, the sink or BS is assumed to be located at the center of the circle. The network area is partitioned into several segments with same angle $\beta$ that is called super-regions. And then, each super region is partitioned into some regions as shown in Fig. 5.

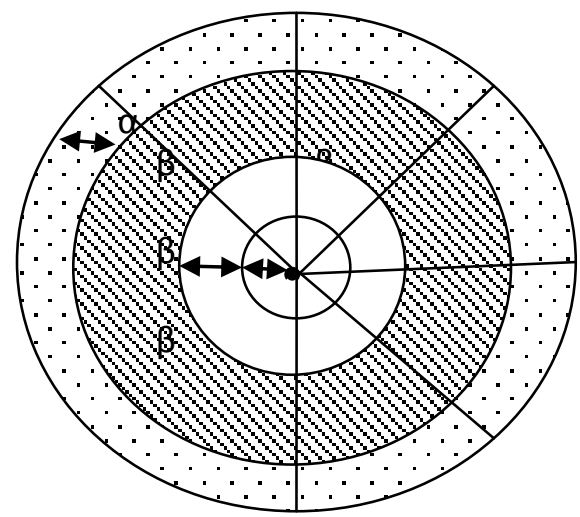

Figure: 4 Circular area-partitioning

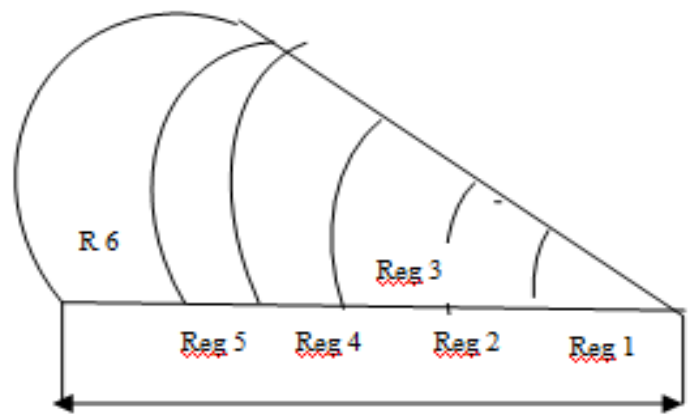

L

Figure: 5 A super-region partitioned into some regions
The goal of the scheme is to obtain load-balancing among region by arranging the width of the regions. The proposed scheme is evaluated to be more superior than the conventional uniform partitioning.

\subsubsection{Balanced Energy-Efficiency (BEE) clustering algorithm}

Most existing clustering algorithms, such as LEACH and HEED, can significantly reduce the power consumption of each sensor node and thus prolong the network lifetime. However, these fail to consider the coverage of the network when evaluating the lifetime of a network. An advanced clustering algorithm should not only manage to extend the longevity, but also maintain the coverage. It is believed that evening the load in every unit area rather than on each single sensor can provide better balanced power usage through the network. Even though, it is hard to achieve perfectly balanced load, the sensors that are still alive should be well distributed over the sensing area. In [15], a novel algorithm based on HEED is provided, named as Balanced Energy-Efficiency (BEE) clustering algorithm. It is shown that BEE exceeds HEED and LEACH in longevity and balanced sensor distribution. The multihop version of BEE is also illustrated in [15], called the Balanced Energy-Efficiency Multihop (BEEM) clustering algorithm, which can further improve the performance of BEE.

\subsubsection{Asynchronous clustering protocol (ACP)}

Most protocols involve periodic network wide reclustering operations that are simultaneously performed, which requires global time synchronization. To address this issue, some recent studies have proposed asynchronous node clustering for networks with direct links from $\mathrm{CHs}$ to the data sink. In [16], an asynchronous node clustering for multihop WSN is proposed, considering dynamic conditions such as residual energy levels and unbalanced data traffic loads caused by packet forwarding. It has been proved that the similar level of lifetime extension can be achieved by reclustering a multihop WSN via independently made decisions at $\mathrm{CHs}$, without a need for time synchronization required by existing synchronization required by existing synchronous protocols.

3.2.6 Self organizing multihop clustering protocol Self organizing WSN protocols are designed to build interconnection links among sensor nodes to make a manageable structure after the initial deployment. During the organization, clustering is a preferred way to achieve scalability. Due to the wireless transmission range and density limitations, connectivity and coverage per cluster can be improved with multihop links. In [17], a self organizing multihop clustering protocol is proposed based on persistent algorithm. Different from the timer based approach, the network decomposition is achieved by iterative local decisions and builds inter-cluster links while initiating new clusters. It provides protocol details and investigated its performance by comparing simulation results with timer based approach when it comes to amount of clusters created, message complexity and configuration time. Even though it has a slightly higher message complexity due to extra messages needed to generate inter-cluster links, the new protocol has a better clustering performance and operates well in low node densities. Configuration is much faster and the system scales well as the network size increases. 


\subsubsection{QoS-oriented events-driven asynchronous clustering protocol}

The quality of service (QoS) to deliver time-and delaysensitive data is critical in events-driven sensor network applications, which are constrained by energy consumption requirements. Most clustering proposals on this problem were based on periodic synchronous approaches, which require time synchronization and are not suitable for events-driven applications. A QoS-oriented events-driven asynchronous clustering protocol is given in [19], which can deliver traffic in a timely and reliable manner. In EEAC, clustering starts asynchronously according to a probability, determined by cluster-head's data transmission rate and residual energy. EEAC avoids time synchronization and adopts composite formula to elect cluster heads. Simulation results have shown that EEAC ensures the real-time transmission of sensitive data, thus reduces the packet loss rate, and evenly distributes nodal energy consumption, and hence prolonging network lifetime.

\subsection{Limitations of Earlier Techniques}

After reviewing the literature, the various limitations that are encountered are stated below:

- Synchronous protocols usually lead to increased complexity time to satisfy more qualitative criteria, whereas important parameters like the number of clusters and the size of each cluster cannot easily be controlled without qualitative cost.

- Additionally, most of these protocols do not apply effective $\mathrm{CHs}$ rotation procedures leading to reduced energy efficiency and worse network lifetime than asynchronous protocols.

- The use of node waiting time to become cluster head has been ignored in most of the existing work.

- In asynchronous clustering technique some nodes will not become cluster head for a longer time even they have more efficiency to become $\mathrm{CH}$.

- The effect of node scalability has been ignored by the majority of researchers

\subsection{Comparison table}

After reviewing the various clustering protocols for WSN, we have made comparison of them on the basis of certain parameters that we have discussed above in this paper.

Table 1: Comparison of Various Clustering Protocols for WSN

\begin{tabular}{|l|l|l|l|l|l|l|l|l|}
\hline $\begin{array}{l}\text { Clustering } \\
\text { Approaches }\end{array}$ & $\begin{array}{l}\text { Cluster } \\
\text { Count }\end{array}$ & $\begin{array}{l}\text { Node } \\
\text { Mobility }\end{array}$ & CH Selection & $\begin{array}{l}\text { In- } \\
\text { cluster } \\
\text { topology }\end{array}$ & $\begin{array}{l}\text { Clustering } \\
\text { Process }\end{array}$ & $\begin{array}{l}\text { CHs } \\
\text { rotation }\end{array}$ & Multilevel & $\begin{array}{l}\text { Time } \\
\text { complexity }\end{array}$ \\
\hline LEACH[6] & Variable & Limited & $\begin{array}{l}\text { Synchronous } \\
\text { Asynchronous }\end{array}$ & 1 hop & Distributed & Yes & No & Constant \\
\hline PEGASIS[7] & Variable & Limited & Synchronous & 1 hop & Distributed & No & No & Variable \\
\hline HGMR[9] & Constant & Yes & Synchronous & K hop & Distributed & No & No & Constant \\
\hline UCMR[11] & Variable & Yes & Synchronous & K hop & Distributed & Yes & Yes & Variable \\
\hline TEEN[8] & Variable & Yes & Synchronous & 1 hop & Distributed & Yes & No & Constant \\
\hline HCC[27] & Variable & Possible & Synchronous & 1 hop & Distributed & No & No & Variable \\
\hline CAWT[28 ] & Variable & No & Synchronous & 2 hop & Distributed & No & No & Constant \\
\hline EACLE[26] & Variable & No & Synchronous & 2 hop & Distributed & Yes & No & Variable \\
\hline ACE[29] & Variable & Possible & Synchronous & K hop & Distributed & No & No & Constant \\
\hline HEED[4] & Variable & Limited & Asynchronous & 1 hop & Distributed & Yes & No & Constant \\
\hline DWEHC[5] & Variable & No & Asynchronous & K hop & Distributed & No & No & Constant \\
\hline CBER[10] & Variable & Limited & Asynchronous & K hop & Distributed & Yes & No & Variable \\
\hline HDO[12] & Variable & Possible & Asynchronous & 1 hop & Distributed & Yes & No & N/A \\
\hline
\end{tabular}




\begin{tabular}{|l|l|l|l|l|l|l|l|l|}
\hline BEEM[15] & Variable & No & Asynchronous & K hop & Distributed & Yes & Yes & Variable \\
\hline ACP[16] & Variable & Yes & Asynchronous & K hop & Distributed & Yes & No & Constant \\
\hline SOMCP[17] & Variable & No & Asynchronous & K hop & Distributed & No & No & Variable \\
\hline SAPSP[18] & Variable & Limited & $\begin{array}{l}\text { Semi } \\
\text { Asynchronous }\end{array}$ & K hop & Distributed & Yes & No & Constant \\
\hline QoS ACP[19] & Variable & Yes & Asynchronous & K hop & Distributed & No & No & Variable \\
\hline DACP[21] & Variable & No & Asynchronous & & Distributed & & & \\
\hline LEACH-C[23] & Variable & Limited & Asynchronous & 1 hop & Centralized & Yes & No & N/A \\
\hline TL-LEACH[6] & Variable & limited & Asynchronous & 1 hop & Distributed & Yes & Yes & Constant \\
\hline
\end{tabular}

\section{CONCLUSIONS}

Network lifetime is crucial in WSN, since recharging or exchanging the sensors is difficult and expensive. Clustering has been widely studied to improve the lifetime of WSN by reducing the number packet transmission. This paper has considered the well-known energy efficient protocols. Furthermore, the combined need for fast convergence time and minimum energy consumption led to appropriate fast distributed asynchronous clustering algorithms which quickly became the most popular and widely used in the field. In these algorithms the nodes are assumed to make fast decisions to become $\mathrm{CHs}$, based on some probability or other local information such as on their residual energy and the desired quality of the final cluster output is considered as a secondary parameter. Another critical feature of many of these algorithms is the periodic reelection of $\mathrm{CHs}$ among all the nodes of the network. Synchronous clustering algorithms that adopt parameters like connectivity, node's proximity, distance, etc., as primary election criteria have also been developed, leading probably to more qualitative output. However the time complexity of these algorithms is more than the leading synchronous/asynchronous clustering algorithms.

Moreover, the extension in multi-hop communication patterns has become necessary as the application of WSNs in real applications becomes larger and larger, whereas the multilevel cluster hierarchies have also been regarded as promising option preserving energy efficiency independent of the growth of the network. Significant progress has also been noted in specialized protocols for timely critical applications i.e. reactive protocols.

The review has shown various challenges in the field of WSNs. However the existing methods have neglected the concept of waiting time to become $\mathrm{CH}$. Moreover the effect of node scalability has also been ignored in the existing research work. So in near future, a new protocol to overcome the limitations of earlier techniques will be proposed.

\section{REFERENCES}

[1] S. Bandyopadhyay and E. Coyle, An energy efficient hierarchical clustering algorithm for wireless sensor networks, in 22nd Annual Joint Conf. of the IEEE Computer and Communications Societies (INFOCOM 2003), San Francisco, CA, April 2003.
[2] Mamalis, Basilis, Damianos Gavalas, Charalampos Konstantopoulos, and Grammati Pantziou. "Clustering in wireless sensor networks." RFID and Sensor Networks: Architectures, Protocols, Security and Integrations, $Y$. Zhang, LT Yang, J. Chen, eds (2009): 324-353.

[3] Guanathillake et.al "Energy Efficient Clustering Algorithm with Global and Local Re-clustering for Wireless Sensor networks" International journal of Elecrical, Robotics, Electronics and Communication Engineering Vol: 7 No. 7, 2013.

[4] Younis, O. Fahmy, S. "HEED: A hybrid, energyefficient, distributed clustering approach for ad-hoc sensor networks". IEEE Trans. Mobile Comput. 2004, 3, 366-379.

[5] 16. Ding, P. Holliday, J. Celik, A. “ Distributed Energy Efficient Hierarchical Clustering for Wireless Sensor Networks" In Proceedings of the 8th IEEE International Conference on Distributed Computing in Sensor Systems (DCOSS), Marina Del Rey, CA, USA, 8-10 June 2005; pp. 322-339.

[6] Loscri, V.; Morabito, G.; Marano, S. A Two-Level Hierarchy for Low-Energy Adaptive Clustering Hierarchy. In Proceedings of the 2nd IEEE Semiannual Vehicular Technology Conference, Dallas, TX, USA, 25-28 September 2005; pp. 1809-1813.

[7] Lindsey, S.; Raghavendra, C.; Sivalingam, K.M. Data gathering algorithms in sensor networks using energy metrics. IEEE Trans. Parallel Distrib. Syst. 2002, 13, 924-935.

[8] 27. Manjeshwar, E.; Agrawal, D.P. TEEN: A Routing Protocol for Enhanced Efficiency in Wireless Sensor Networks. In Proceedings of the 15th International Parallel and Distributed Processing Symposium (IPDPS), San Francisco, CA, USA, 23-27 April 2001; pp. 2009-2015.

[9] Koutsonikola, D.; Das, S.; Charlie, H.Y.; Stojmenovic, I. Hierarchical geographic multicast routing for wireless sensor networks. Wirel. Netw. 2010, 16, 449-466.

[10] Sidhik, A. Sharma, U. Hernandez-Jayo, and N. Sainz "A Novel Cluster-based Energy Efficient Routing in Wireless Sensor Networks" IEEE 27th International 
Conference on Advanced Information Networking and Applications, 2013.

[11] Hari, U., B. Ramachandran, and Chris Johnson. "An Unequally Clustered Multihop Routing protocol for Wireless Sensor Networks." In Advances in Computing, Communications and Informatics (ICACCI), 2013 International Conference on, pp. 1007-1011. IEEE, 2013.

[12] Suharjono, Amin, and Gamantyo Hendrantoro. "Hop distances optimization for balancing the energy consumption of multi-hop clustered Wireless sensor Networks." In Computer, Control, Informatics and Its Applications (IC3INA), 2013 International Conference on, pp. 49-52. IEEE, 2013.

[13] Khediri, Salim EL, Nejah Nasri, Anne Wei, and Abdennaceur Kachouri. "A New Approach for Clustering in Wireless Sensors Networks Based on LEACH."Procedia Computer Science 32 (2014): 11801185.

[14] Xu, Lina, M. J. O'Grady, G. M. P. O'Hare, and Rem Collier. "Reliable multihop intra-cluster communication for Wireless Sensor Networks." In Computing, Networking and Communications (ICNC), 2014 International Conference on, pp. 858-863. IEEE, 2014.

[15] Xu, Lina, G. M. P. O'Hare, and Rem Collier. "A Balanced Energy-Efficient Multihop clustering scheme for Wireless Sensor Networks." In Wireless and Mobile Networking Conference (WMNC), 2014 7th IFIP, pp. 18. IEEE, 2014.

[16] Vural, Serdar, PirabakaranNavaratnam, Ning Wang, and Rahim Tafazolli. "Asynchronous clustering of multihop Wireless Sensor Networks." In Communications (ICC), 2014 IEEE International Conference on, pp. 472-477. IEEE, 2014.

[17] Saglam, O., and M. E. Dalkili. "A self organizing multihop clustering protocol for wireless sensor networks." In Mobile Ad-hoc and Sensor Networks, 2009. MSN'09. 5th International Conference on, pp. 3340. IEEE, 2009.

[18] Hsu, Chih-Shun, and Yu-Chee Tseng. "Cluster-based semi-asynchronous power-saving protocols for multi-hop ad hoc networks." In Communications, 2005. ICC 2005. 2005 IEEE International Conference on, vol. 5, pp. 3166-3170. IEEE, 2005.

[19] Luo, Juan, Hui-zhong Liu, Ren-fa Li, and Lichun Bao. "QoS-oriented asynchronous clustering protocol in wireless sensor networks." In Wireless Communications,
Networking and Mobile Computing, 2008. WiCOM'08. 4th International Conference on, pp. 1-4. IEEE, 2008.

[20] Hebden, Peter, and Adrian R. Pearce. "Distributed asynchronous clustering for self-organisation of wireless sensor networks." In Intelligent Sensing and Information Processing, 2006. ICISIP 2006. Fourth International Conference on, pp. 37-42. IEEE, 2006.

[21] Wendi Beth Heinzelman, "Application-Specific Protocol Architectures for Wireless Network," Department of Electrical Engineering and Computer Science, Massachusetts Institute of Technology, 2000.

[22] Wendi B. Heinzelman," An Application-Specific Protocol Architecture for Wireless Microsensor Networks," IEEE Transactions on Wireless Communications, vol. 1, no. 4, october 2002.

[23] Xiangning, Fan, and Song Yulin. "Improvement on LEACH protocol of wireless sensor network." In Sensor Technologies and Applications, 2007. SensorComm 2007. International Conference on, pp. 260-264. IEEE, 2007.

[24] M. BaniYassein, A. Al-zou'bi, Y. Khamayseh, W.Mardini, " An Improvement on LEACH Protocol of Wireless Sensor Network (VLEACH)," International Journal of Digital Content Technology and its Applications Volume 3, Number 2, June 2009.

[25] Wairagu G. Richard, "Extending LEACH routing algorithm for Wireless Sensor Network," Data Communications Engineering, Makerere University, 2009.

[26] K. Yanagihara, J. Taketsugu, K. Fukui, S. Fukunaga, S. Hara and K.I. Kitayama, EACLE: Energy-aware clustering scheme with transmission power control for sensor networks, Springer, Wireless Personal Communications, 40(3), 401-415, 2007.

[27] S. Banerjee and S. Khuller, A clustering scheme for hierarchical control in multi-hop wireless networks, in Proceedings of 20th Joint Conference of the IEEE Computer and Communications Societies (INFOCOMS 01), Anchorage, AK, April 2001.

[28] Wen and W. Sethares, Automatic decentralized clustering for WSNs, EURASIP Journal on Wireless Communications and Networking, 5(5), 686-697, 2005.

[29] H. Chan and A. Perrig, ACE: an emergent algorithm for highly uniform cluster formation, in Proceedings of the 1st European Workshop on Sensor Networks (EWSN), Berlin, Germany, January 2004. 